\title{
In response to Fogarty et al. and why adjuvant whole brain radiotherapy is not recommended routinely
}

\author{
Mark B. Pinkham ${ }^{1,2^{*}}$, Arjun Sahgal ${ }^{3}$, Andrew P. Pullar ${ }^{1,4}$ and Matthew C. Foote ${ }^{1,2}$
}

\begin{abstract}
The routine use of adjuvant whole brain radiotherapy (AWBRT) after surgery or stereotactic radiosurgery is now discouraged by a number of international expert panels. Three decades of randomised studies have shown that, although AWBRT improves radiological measures of intracranial disease control, the clinical benefit is unclear and it is also associated with inferior quality of life and neurocognitive function. The number of patients with melanoma in these trials was low, but data suggesting that treatment-related side effects should vary according to histology of the primary malignancy are lacking. For metastatic melanoma, the role of AWBRT to control microscopic disease in the brain is also a less relevant concern because systemic therapies with intracranial activity are now available. Whether AWBRT is useful in select patients deemed at high risk of neurologic death remains undefined.
\end{abstract}

Keywords: Brain metastases, Melanoma, Radiosurgery, Gamma knife, Whole brain radiotherapy

We read with interest the article by Fogarty et al. debating the role of adjuvant whole brain radiotherapy (AWBRT) after surgery or stereotactic radiosurgery (SRS) for brain metastases [1]. They propose that withholding AWBRT 'may not be in the best interests of patients, nor of health systems' and that the American Society of Radiation Oncology (ASTRO) should retract its recommendation to discourage the routine use of AWBRT in this setting [2].

The management of any patient with incurable disease should focus on maximising quality and quantity of survival. Local therapy without AWBRT is now a standard treatment for patients with limited number of brain metastases and a more favourable prognosis. This recommendation is endorsed by multiple expert panels including ASTRO, National Cancer Comprehensive Network (NCCN) and the European Association of Neuro-Oncology (EANO) [3-5]. Nearly three decades of randomised studies have consistently shown that the omission of AWBRT after surgery or SRS is not associated with inferior survival or functional independence

\footnotetext{
* Correspondence: Mark.pinkham@trinity.oxon.org

${ }^{1}$ Department of Radiation Oncology, Princess Alexandra Hospital, Brisbane, Queensland 4102, Australia

${ }^{2}$ University of Queensland, Brisbane, Queensland, Australia

Full list of author information is available at the end of the article
}

[6-11]. Although AWBRT improves radiological measures of intracranial disease control, this is of uncertain clinical benefit because most patients die from extracranial disease and AWBRT adversely affects quality of life (QoL) and neurocognitive function (NCF) $[8,11,12]$. Despite an increased need for radiological surveillance and salvage therapy at recurrence, a number of North American studies have concluded that SRS alone provides cost-effective care compared to SRS with AWBRT [13-15].

Many patients will experience acute side effects during or shortly after AWBRT including dermatitis, alopecia and fatigue. Neurotoxicity is traditionally said to be a late effect only observed in a minority of long-term survivors. However this is inaccurate because deficits in NCF can occur within 3-6 months from treatment $[8,11,16-18]$. NCF is important because it can predict QoL [19], functional independence and survival [16, 20]. Declining NCF increases caregiver burden [21] and impairs financial, work and social activities [22, 23]. Although NCF was not assessed in the largest randomised study of AWBRT ever performed, patient-reported cognitive function was analysed [9, 12]. In 341 patients randomised to observation or AWBRT after SRS or surgery to 1-3 brain metastases, AWBRT was associated with 
significantly lower mean test scores at 8 weeks and 12 months. AWBRT also resulted in inferior healthrelated QoL relating to fatigue, physical functioning and role functioning at various time points. The Alliance randomised study presented at American Society of Clinical Oncology (ASCO) 2015 mentioned by Fogarty et al. has now been published [11]. This trial is noteworthy because the primary endpoint was NCF at 3 months. Out of 213 participants with 1-3 brain metastases, deterioration in at least one NCF test was noted in $64 \%$ of patients receiving SRS and 92\% receiving SRS with AWBRT. Statistically significant deteriorations were seen in immediate memory (8\% versus $30 \%)$, delayed memory (20\% versus $51 \%$ ) and verbal fluency (2\% versus $19 \%)$. Overall QoL (mean change from baseline -0.1 versus -12 points) and functional well-being (2.5 versus -22.3 points) were also superior at 3 months for SRS alone. Such differences persisted in the subset of 34 evaluable patients (16\%) surviving at least 12 months from randomisation and reached statistical significance at various time points. These findings are consistent with a similar but smaller study by Chang et al. [8], which was potentially confounded by an unexpected imbalance in median survival between the two arms. Therefore, although AWBRT reduces the rate of intracranial failure, overall its deleterious effects on NCF are greater in the majority of patients than the deleterious effects of recurrence itself. Data to suggest that this treatment-related effect should vary according to histology of the primary malignancy is lacking.

Fogarty et al. state that AWBRT avoids a neurologic death. This was shown in two randomised studies $[6,9]$ but not confirmed in the others $[7,8]$. In the metaanalysis by Sahgal et al., the risk of neurologic death with AWBRT was reduced from $30 \%$ to $25 \%$ [24]. Given the potential toxicities described, whether AWBRT is justified in up to 20 patients to prevent one additional neurologic death is uncertain. For many clinicians, this uncertainty has grown with the emergence of increasingly effective systemic therapies exhibiting potential intracranial activity. Consistent with this change, a more contemporary multi-institutional prospective series of 1194 select patients with up to 10 brain metastases treated with Gamma Knife SRS without AWBRT reported a neurologic death rate of only $8 \%$ [25].
We applaud the investigators of the ANZMTG 01.07 WBRTMel trial for their dogged determination to complete accrual. The trial hypothesis is that the addition of AWBRT will improve intracranial control and survival without significant impairment of QoL or NCF. The primary endpoint is distant intracranial control at 12 months and the protocol has been amended to permit AWBRT with hippocampal avoidance. Although Fogarty et al. believe that the ASTRO recommendation is negatively impacting on accrual to this trial, it is possible other factors have also influenced the willingness of patients and physicians to recruit to this study. Whether clinical equipoise truly remains or not because melanoma-histology specific data is sparse, a major concern remains the clinical relevance of the trial primary endpoint which is a radiological measure of disease. Interpretability of the data may also be limited because stratification for systemic therapy utilisation is lacking. Since this trial was designed, a number of targeted and immunotherapy systemic agents have become widely available for metastatic melanoma. Many are known to have activity in the brain $[26,27]$ although potential effects on QoL, fatigue and NCF are not well described.

Various subgroup analyses and retrospective series might suggest subgroups of patients that could benefit from AWBRT, but reports are contradictory [28-30] and high quality data is lacking. The effects of AWBRT on intracranial disease control, QoL and NCF are now clearly defined in an unselected population. Whether AWBRT can be used to control microscopic or occult melanoma in the brain is a less relevant concern nowadays because systemic therapy can be used. The role of hippocampal sparing AWBRT is yet to be defined in any population and the ASTRO recommendation should not preclude the proper assessment of new technologies in appropriately selected patients. However, optimal sequencing of treatment modalities is now a more relevant area of investigation rather than the effect of SRS and/or AWBRT alone. In this context, mixed-histology trials without stratification are no longer appropriate and enrichment for certain molecular subtypes are required. Multicentre efforts will be needed to permit statistically sound results within a reasonable time frame, thus providing data that remains useful and relevant to patients and their doctors.

\section{In reply to Pinkham et al.}

Gerald B. Fogarty ${ }^{1,2,3,4,0^{*}}$, Angela Hong ${ }^{1,2,3}$, Vinai Gondi ${ }^{7,8}$, Bryan Burmeister ${ }^{3,4,5}$, Kari Jacobsen ${ }^{6}$, Serigne Lo ${ }^{1,2,3}$, Elizabeth Paton ${ }^{2,3}$, Brindha Shivalingam ${ }^{9}$ and John F. Thompson 1,2,3

\footnotetext{
${ }^{1}$ Melanoma Institute Australia, Poche Centre, North Sydney, Australia

${ }^{2}$ Sydney Medical School, The University of Sydney, Sydney, Australia
} 


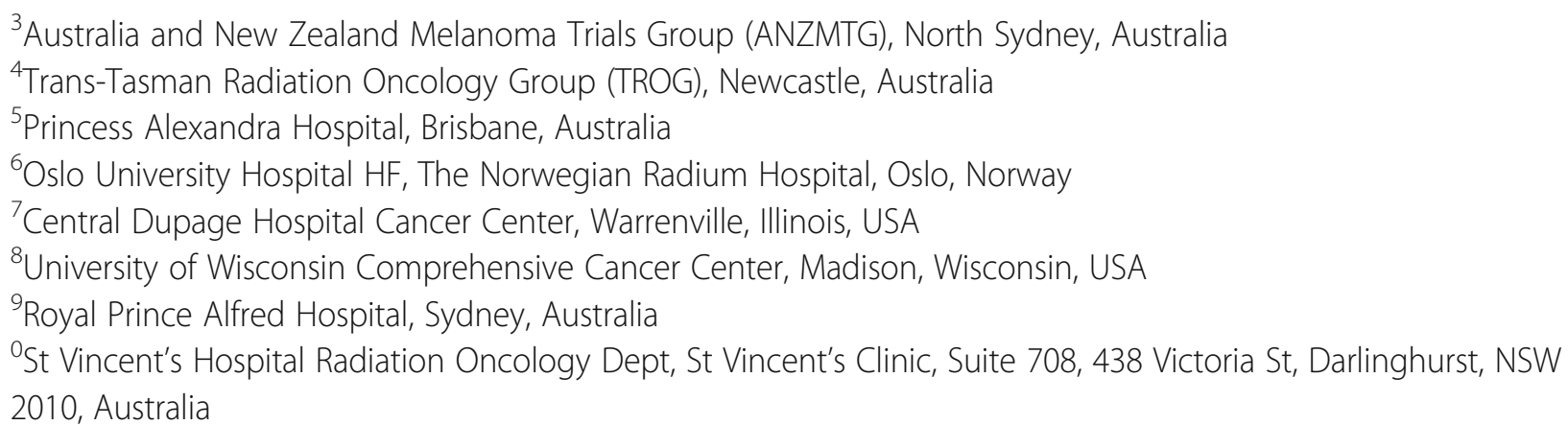

We thank Pinkham et al. for their reply to our article [1]. In many areas we are in agreement. For example, we agree that adjuvant whole brain radiotherapy (AWBRT) without hippocampal sparing administered to patients with brain metastases can cause side effects including adverse effects on quality of life (QoL) and neurocognitive function (NCF). We also accept the evidence that most patients die from extracranial disease so that the impact of any radiotherapy (RT) to the brain should be measured in terms of palliative endpoints and not overall survival.

The thrust of our article, however, has been missed. To repeat it - even though 170,000 patients are diagnosed with brain metastases (BMs) in the United States every year, and are therefore possible candidates for studies assessing the value of AWBRT, in three decades only 774 patients had been enrolled in randomised controlled trials (RCTs) before the publishing of the American Society for Radiation Oncology (ASTRO) Item 5 of its "Choosing Wisely 2014" list. This recommended: "Don't routinely add adjuvant whole brain radiation therapy to SRS for limited brain metastases" [2].

Our experience has been that the ASTRO guideline has had a significant negative impact on accrual to the internationally accruing ANZMTG 01.07 WBRTMel trial [31]. This is an RCT investigating AWBRT following local treatment (surgery or stereotactic radiosurgery) in patients with one to three BMs from melanoma - one of the histologies that has consistently shown high rates of distant brain failure [28, 32, 33], and thus most likely to benefit from WBRT. This trial is the only single histology AWBRT trial so far attempted. To date WBRTMel has accrued 214 of a required 220 patients but recently accrual has diminished dramatically. Several aspects of this trial had already been reported prior to the publication of ASTRO guideline [31, 32]. Dr. Pinkham's institution, from which 29 patients had been randomised to the WBRTMel trial, has not accrued a single patient since the guideline was published. The guideline should at least have added a caveat "except in the case of a clinical trial".
There is an urgent need for more data, especially when a phase two trial investigating hippocampal-avoiding AWBRT (HAWBRT) showed significantly less NCF decline compared to historical controls [34]. We now need more randomised data to confirm the benefit of HAWBRT. It is important to note that hippocampal avoidance is allowed on WBRTMel and over 22 cases have had this form of treatment.

If the WBRTMel trial shows AWBRT in melanoma to be useless, then we will be amongst the first to recommend that it be abandoned. However, we contend that the procedure should only be abandoned only on the basis of adequate level one clinical trial evidence. We contend that the accumulation of more data is a wiser choice than abandoning AWBRT before conclusive RCT evidence is available, especially in melanoma.

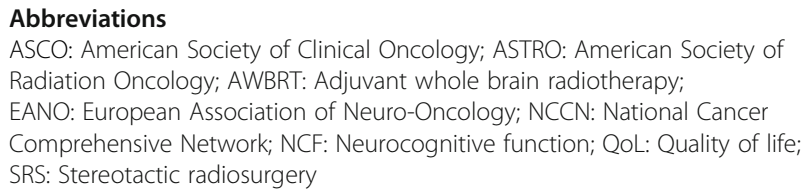

Funding

The authors declare no funding was sourced for this manuscript.

\section{Availability of data and materials \\ Not applicable}

\section{Authors' contributions}

MBP drafted initial manuscript. All authors provided comments and edits. All authors read and approved final manuscript.

\section{Authors' information}

Not applicable

Ethics approval and consent to participate

Not applicable

Consent for publication

Not applicable

Competing interests

The authors declare that they have no competing interests. 


\section{Publisher's Note}

Springer Nature remains neutral with regard to jurisdictional claims in published maps and institutional affiliations.

\section{Author details}

'Department of Radiation Oncology, Princess Alexandra Hospital, Brisbane, Queensland 4102, Australia. ${ }^{2}$ University of Queensland, Brisbane, Queensland, Australia. ${ }^{3}$ Department of Radiation Oncology, Sunnybrook Health Sciences Centre, University of Toronto, Toronto, Ontario, Canada. ${ }^{4}$ Queensland University of Technology, Brisbane, Queensland, Australia.

Received: 28 November 2016 Accepted: 4 October 2017 Published online: 15 November 2017

\section{References}

1. Fogarty GB, Hong A, Gondi V, Burmeister B, Jacobsen K, Lo S, et al. Debate: adjuvant whole brain radiotherapy or not? More data is the wiser choice. BMC Cancer. 2016;16:372

2. Choosing wisely. ASTRO releases second list of five radiation oncology treatments to question, as part of national choosing wisely ${ }^{\oplus}$ campaign. [Internet]. [cited 2015 Mar 12]. Available from: http://www.choosingwisely. org/astro-releases-second-list/.

3. Tsao MN, Rades D, Wirth A, Lo SS, Danielson BL, Gaspar LE, et al. Radiotherapeutic and surgical management for newly diagnosed brain metastasis(es): an American Society for Radiation Oncology evidence-based guideline. Pract Radiat Oncol. 2012;2:210-25.

4. National Comprehensive Cancer Network (NCCN) clinical practice guidelines in oncology (NCCN guidelines ${ }^{\oplus}$ ) central nervous system cancers version 1 2014 [Internet]. [cited 2014 Jun 30]. Available from: http://www.nccn.org/ professionals/physician_gls/pdf/cns.pdf.

5. Soffietti R, Abacioglu U, Baumert B, Combs SE, Kinhult S, Kros JM, et al. Diagnosis and treatment of brain metastases from solid tumors: guidelines from the European Association of Neuro-Oncology (EANO). Neuro-Oncol. 2017;19:162-74.

6. Patchell RA, Tibbs PA, Regine WF, Dempsey RJ, Mohiuddin M, Kryscio RJ, et al. Postoperative radiotherapy in the treatment of single metastases to the brain: a randomized trial. JAMA J Am Med Assoc. 1998:280:1485-9.

7. Aoyama H, Shirato H, Tago M, Nakagawa K, Toyoda T, Hatano K, et al. Stereotactic radiosurgery plus whole-brain radiation therapy vs stereotactic radiosurgery alone for treatment of brain metastases: a randomized controlled trial. JAMA J Am Med Assoc. 2006:295:2483-91.

8. Chang EL, Wefel JS, Hess KR, Allen PK, Lang FF, Kornguth DG, et al. Neurocognition in patients with brain metastases treated with radiosurgery or radiosurgery plus whole-brain irradiation: a randomised controlled trial. Lancet Oncol. 2009;10:1037-44.

9. Kocher M, Soffietti R, Abacioglu U, Villà S, Fauchon F, Baumert BG, et al. Adjuvant whole-brain radiotherapy versus observation after radiosurgery or surgical resection of one to three cerebral metastases: results of the EORTC 22952-26001 study. J Clin Oncol Off J Am Soc Clin Oncol. 2011;29:134-41.

10. Tsao MN, Lloyd N, Wong RKS, Chow E, Rakovitch E, Laperriere N, et al. Whole brain radiotherapy for the treatment of newly diagnosed multiple brain metastases. Cochrane Database Syst Rev Online. 2012;4:CD003869.

11. Brown PD, Jaeckle K, Ballman KV, Farace E, Cerhan JH, Anderson SK, et al. Effect of Radiosurgery alone vs Radiosurgery with whole brain radiation therapy on cognitive function in patients with 1 to 3 brain metastases: a randomized clinical trial. JAMA. 2016:316:401-9.

12. Soffietti R, Kocher M, Abacioglu UM, Villa S, Fauchon F, Baumert BG, et al. A European Organisation for Research and Treatment of Cancer phase III trial of adjuvant whole-brain radiotherapy versus observation in patients with one to three brain metastases from solid tumors after surgical resection or radiosurgery: quality-of-life results. J Clin Oncol Off J Am Soc Clin Oncologia. 2013:31:65-72.

13. Hall MD, McGee JL, McGee MC, Hall KA, Neils DM, Klopfenstein JD, et al. Cost-effectiveness of stereotactic radiosurgery with and without whole-brain radiotherapy for the treatment of newly diagnosed brain metastases. J Neurosurg. 2014;121(Suppl):84-90.

14. Lal LS, Byfield SD, Chang EL, Franzini L, Miller L-A, Arbuckle R, et al. Cost-effectiveness analysis of a randomized study comparing radiosurgery with radiosurgery and whole brain radiation therapy in patients with 1 to 3 brain metastases. Am J Clin Oncol. 2012;35:45-50.
15. Lester SC, Taksler GB, Kuremsky JG, Lucas JT, Ayala-Peacock DN, Randolph DM, et al. Clinical and economic outcomes of patients with brain metastases based on symptoms: an argument for routine brain screening of those treated with upfront radiosurgery. Cancer. 2014;120:433-41.

16. Meyers CA, Smith JA, Bezjak A, Mehta MP, Liebmann J, Illidge T, et al. Neurocognitive function and progression in patients with brain metastases treated with whole-brain radiation and motexafin gadolinium: results of a randomized phase III trial. J Clin Oncol Off J Am Soc Clin Oncol. 2004;22:157-65.

17. Sun A, Bae K, Gore EM, Movsas B, Wong SJ, Meyers CA, et al. Phase III trial of prophylactic cranial irradiation compared with observation in patients with locally advanced non-small-cell lung cancer: neurocognitive and quality-oflife analysis. J Clin Oncol Off J Am Soc Clin Oncol. 2011;29:279-86.

18. Brown PD, Pugh S, Laack NN, Wefel JS, Khuntia D, Meyers C, et al. Memantine for the prevention of cognitive dysfunction in patients receiving whole-brain radiotherapy: a randomized, double-blind, placebo-controlled trial. Neuro-Oncol. 2013;15:1429-37.

19. Li J, Bentzen SM, Li J, Renschler M, Mehta MP. Relationship between neurocognitive function and quality of life after whole-brain radiotherapy in patients with brain metastasis. Int J Radiat Oncol Biol Phys. 2008;71:64-70.

20. Corn BW, Moughan J, Knisely JPS, Fox SW, Chakravarti A, Yung WKA, et al. Prospective evaluation of quality of life and neurocognitive effects in patients with multiple brain metastases receiving whole-brain radiotherapy with or without thalidomide on radiation therapy oncology group (RTOG) trial 0118. Int J Radiat Oncol Biol Phys. 2008;71:71-8.

21. Germain S, Adam S, Olivier C, Cash H, Ousset PJ, Andrieu S, et al. Does cognitive impairment influence burden in caregivers of patients with Alzheimer's disease? J Alzheimers Dis JAD. 2009;17:105-14.

22. Griffith HR, Belue K, Sicola A, Krzywanski S, Zamrini E, Harrell L, et al. Impaired financial abilities in mild cognitive impairment: a direct assessment approach. Neurology. 2003;60:449-57.

23. Meyers CA. Functional outcomes. In: Berger MS, Prados M, editors. Textbook of Neuro-oncology. Philadelphia: Elsevier; 2005. p. 101-4.

24. Sahgal A, Aoyama H, Kocher M, Neupane B, Collette S, Tago M, et al. Phase 3 trials of stereotactic Radiosurgery with or without whole-brain radiation therapy for 1 to 4 brain metastases: individual patient data meta-analysis. Int J Radiat Oncol. 2015:91:710-7.

25. Yamamoto M, Serizawa T, Shuto T, Akabane A, Higuchi Y, Kawagishi J, et al. Stereotactic radiosurgery for patients with multiple brain metastases (JLGK0901): a multi-institutional prospective observational study. Lancet Oncol. 2014;15:387-95.

26. Goyal S, Silk AW, Tian S, Mehnert J, Danish S, Ranjan S, et al. Clinical Management of Multiple Melanoma Brain Metastases: a systematic review. JAMA Oncol. 2015:1:668-76.

27. Ajithkumar T, Parkinson C, Fife K, Corrie P, Jefferies S. Evolving treatment options for melanoma brain metastases. Lancet Oncol. 2015;16:e486-97.

28. Ayala-Peacock DN, Peiffer AM, Lucas JT, Isom S, Kuremsky JG, Urbanic JJ, et al. A nomogram for predicting distant brain failure in patients treated with gamma knife stereotactic radiosurgery without whole brain radiotherapy. Neuro-Oncol. 2014;16:1283-8.

29. Chen X, Xiao J, Li X, Jiang X, Zhang Y, Xu Y, et al. Risk factors of distant brain failure for patients with newly diagnosed brain metastases treated with stereotactic radiotherapy alone. Radiat Oncol Lond Engl. 2011;6:175.

30. Press RH, Prabhu RS, Nickleach DC, Liu Y, Shu H-KG, Kandula S, et al. Novel risk stratification score for predicting early distant brain failure and salvage whole-brain radiotherapy after stereotactic radiosurgery for brain metastases. Cancer. 2015:121:3836-43.

31. Fogarty G, Morton RL, Vardy J, et al. Whole brain radiotherapy after local treatment of brain metastases in melanoma patients-a randomised phase III trial. BMC Cancer. 2011;11:142.

32. Sawrie SM, Guthrie BL, Spencer SA, et al. Predictors of distant brain recurrence for patients with newly diagnosed brain metastases treated with stereotactic radiosurgery alone. Int J Radiat Oncol Biol Phys. 2008;70(1):181-6.

33. Farris M, MCTyre ER, Cramer CK, et al. Brain metastasis velocity: a novel prognostic metric predictive of overall survival and freedom from whole-brain radiation therapy after distant brain failure following upfront Radiosurgery alone. Int J Radiat Oncol Biol Phys. 2017;98(1):131-41.

34. Gondi V, Pugh SL, Tome WA, et al. Preservation of memory with conformal avoidance of the hippocampal neural stem-cell compartment during whole-brain radiotherapy for brain metastases (RTOG 0933): a phase II multi-institutional trial. J Clin Oncol. 2014;32(34):3810-6. 